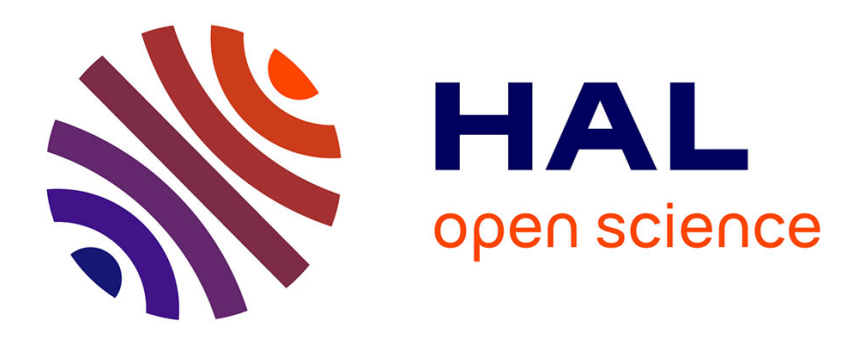

\title{
Poisson approximation for some point processes in reliability
}

Jean-Bernard Gravereaux, James Ledoux

\section{To cite this version:}

Jean-Bernard Gravereaux, James Ledoux. Poisson approximation for some point processes in reliability. Advances in Applied Probability, 2004, 36 (2), pp.455-470. hal-00837505

\section{HAL Id: hal-00837505 \\ https://hal.science/hal-00837505}

Submitted on 19 Aug 2013

HAL is a multi-disciplinary open access archive for the deposit and dissemination of scientific research documents, whether they are published or not. The documents may come from teaching and research institutions in France or abroad, or from public or private research centers.
L'archive ouverte pluridisciplinaire HAL, est destinée au dépôt et à la diffusion de documents scientifiques de niveau recherche, publiés ou non, émanant des établissements d'enseignement et de recherche français ou étrangers, des laboratoires publics ou privés. 


\title{
Poisson approximation for some point processes in reliability
}

\author{
J.-B. Gravereaux and James Ledoux*
}

11 February 2004

\begin{abstract}
In this paper, we consider a failure point process related to the Markovian Arrival Process defined by Neuts. We show that it converges in distribution to a homogeneous Poisson process. This convergence takes place in context of rare occurrences of failures. We also provide a convergence rate of the convergence in total variation of this point process using an approach developed by Kabanov, Liptser and Shiryaev for the doubly-stochastic Poisson process driven by a finite Markov process.
\end{abstract}

Keywords: Compensator, Software reliability, Markovian Arrival Process, Doubly Stochastic Process

AMS : 60G55; 60J25; 60J75; 90B25; 93E11

\section{Introduction}

This work originates in Littlewood papers [12],[13] on a Markov-type model for reliability assessment of a modular software. Basically, for a software with a finite number of modules :

- the structure of the software is represented by a finite continuous time Markov chain (CTMC) $\left(X_{t}\right)_{t}$ where $X_{t}$ is the active module at time $t$;

- when module $i$ is active, failures times are part of a homogeneous Poisson Process (HPP) with intensity $\mu(i)$;

- when control switches from module $i$ to module $j$ a failure may happen with probability $\mu(i, j)$;

*IRMAR UMR 6625 \& INSA, 20 avenue des Buttes de Coësmes, 35708 Rennes Cedex 7, France 
- when any failure appears, it does not affect the software because the execution is assumed to be restarted instantaneously. Such event is referred as to a secondary failure in [9].

Extend of such a model is considered in [9], taking into account the influence of failures on the execution dynamic of the software and dealing with the delays in recovering an operational state. Transient analysis was provided by means of results from [10]. Roughly speaking, failure point process was a Markovian Arrival Process (MAP) as defined by Neuts (see e.g. [15]). Therefore, it is well known that we obtain as particular instances of our failure process: a phase-type renewal process, a doubly stochastic poisson process with a stochastic intensity driven by a CTMC (also called a Markov Modulated Poisson Process (MMPP) in queueing literature), etc.

An important issue in reliability theory, specifically for software systems, is what happens when the failure parameters tend to be smaller and smaller. Littlewood stated in [12] (or in [13] for a semi-Markov process $\left(X_{t}\right)$ )

As all failure parameters $\mu(i), \mu(i, j)$ tend to zero, the failure process described above is asymptotically an HPP with intensity

$$
\lambda=\sum_{i} \pi(i)\left[\sum_{j \neq i} Q(i, j) \mu(i, j)+\mu(i)\right]
$$

where $\pi$ and $Q$ are the stationary distribution and the generator of the CTMC $\left(X_{t}\right)$ (assumed to be irreducible), respectively. This statement is well-known in the community of software reliability and has widely supported the hierarchical approach for modeling modular software (see e.g. [6] for details). However, to the best of our knowledge, no proof of this fact is reported in the applied probability literature. The aim of this note is to provide precise statements and proofs for the asymptotic of the general failure point process (pp) defined in [9] for which the Littlewood's model is a particular case. Specifically, we show that the counting process corresponding to this pp converges in distribution to the counting process of an HPP when failure parameters tend to zero but at a specific time scale. Roughly speaking, we introduce a small parameter $\varepsilon$ in the failure parameters and the convergence takes place at time scale $t / \varepsilon$ (in other words when failure parameters are small and on a large horizon time). Proving this result is easy using a criterion of convergence in distribution given in [8] for instance. It is based on the convergence in probability of compensators corresponding to the various counting processes. In fact, the counting process converges in variation to the counting process of an HPP and the convergence rate will be stated using a method developed in [8], for the MMPP.

Note that the class of MMPP is widely used as a model of traffic streams for communication systems. It is easily seen that dealing with the present issue is equivalent to consider asymptotic for MMPP with a fast modulating Markov chain. Thus we retrieve the more or less known fact that, when jitterness takes place, the arrival process tends to Poissonian (see [14, page 116] for a partial discussion). 
Paper is organized as follows. Section 2 recalls some background on the pp studied here. Moreover, the compensator of the $\mathrm{pp}$ is derived in a straightforward manner. In Section 3, we report results about convergence in distribution of the pp to an HPP. Connection to problem of fast modulation in the case of an MMPP is briefly addressed in Subsection 3.3. Rate of convergence in total variation of the pp is stated in Section 4 . Appendix A recalls some estimate of convergence rate of singularly perturbated generator provided in [18]. The derivation of an inequality used in the text is reported in Appendix B.

\section{Definition of the counting process. Compensator}

\subsection{Definition of the model}

We do not report the rationale underlying the definition of the reliability model discussed here. We refer to [9] for details. We just need of its mathematical formulation.

Parameters $\mu(\cdot, \cdot), \mu(\cdot)$ are as in Introduction. These failures was called secondary events in [9]. Process $X=\left(X_{t}\right)_{t}$ is an irreducible CTMC with infinitesimal generator $Q=(Q(i, j))_{i, j \in \mathcal{M}}$ where $\mathcal{M}$ is the finite set $\{1, \ldots, M\} . \quad X_{t}$ is the active component at time $t$ for a failure-free system, that is $X$ is the execution process. Vector $\alpha=(\alpha(i))_{i \in \mathcal{M}}$ denotes the distribution of random variable $X_{0}$. New parameters $\lambda(i, j)$ and $\lambda(i)(i, j \in \mathcal{M})$ are introduced with the same meaning than $\mu(i, j)$ and $\mu(i)$. But when such a type of failure happens in module $i$ or during a transition from module $i$, there is a probability $p(i, k)$ that execution restarts in module $k$. So that, for each $i \in \mathcal{M}$, $(p(i, k))_{k \in \mathcal{M}}$ is a probability distribution. Such event was referred as to a primary failure in [9]. Simultaneous occurrence of a primary and secondary events is neglected. For simplicity, we do not consider delay in recovering an operational state as used in [9].

Then taking into account failure occurrences, random variable $X_{t}^{*}$ gives the active component at time $t$. The random variable $N_{t}$ counts the number of (primary and secondary) failures in interval $] 0, t](N(0)=0)$. Thus, $N=\left(N_{t}\right)_{t}$ is the counting process of the failure pp. Under the various assumptions in [9], the bivariate process $Z:=\left(N_{t}, X_{t}^{*}\right)_{t}$ can be considered as a CTMC over the state space $S=\mathbb{N} \times \mathcal{M}$. Its infinitesimal generator, denoted by $G$, has the special structure

$$
G:=\left(\begin{array}{cccc}
D_{0} & D_{1} & 0 & \cdots \\
0 & D_{0} & D_{1} & \ddots \\
\vdots & \ddots & \ddots & \ddots
\end{array}\right)
$$

using a lexicographic order on state space $S$. Matrices $D_{0}$ and $D_{1}$ are defined by

$$
\begin{aligned}
D_{0}(i, j) & :=Q(i, j)(1-\lambda(i, j))(1-\mu(i, j)) \text { if } i \neq j, \\
D_{0}(i, i) & :=-\sum_{j \neq i} Q(i, j)-\lambda(i)-\mu(i)
\end{aligned}
$$




$$
\begin{aligned}
D_{1}(i, j) & :=\left[\lambda(i)+\sum_{k \neq i} Q(i, k) \lambda(i, k)\right] p(i, j)+Q(i, j)[1-\lambda(i, j)] \mu(i, j) \text { if } i \neq j, \\
D_{1}(i, i) & :=\left[\lambda(i)+\sum_{k \neq i} Q(i, k) \lambda(i, k)\right] p(i, i)+\mu(i) .
\end{aligned}
$$

Note that $\max _{x}|G(x, x)|<+\infty$. The structure of the generator $G$ shows that $N$ is the counting process of a MAP. Finally, $X^{*}$ is a CTMC with state space $\mathcal{M}$, initial distribution $\alpha$ and generator

$$
Q^{*}:=D_{0}+D_{1}
$$

$X^{*}$ is supposed to be right continuous with left limits (c.a.d-l.a.g.). If the failure parameters are assumed to be such that $\lambda(i, j)<1$ for any $(i, j)$, then $X^{*}$ is irreducible since $X$ is. This assumption is not very stringent.

\section{Example 2.1 (Littlewood's model)}

Assume that there is no primary failures, that is, $\lambda(i)=0$ and $\lambda(i, j)=0$ for all $i, j \in \mathcal{M}$. We obtain the model of Littlewood. Then matrices $D_{0}$ and $D_{1}$ are given by

$$
D_{0}(i, j)=\left\{\begin{array}{cl}
Q(i, j)(1-\mu(i, j)) & \text { if } i \neq j, \\
-\sum_{j \neq i} Q(i, j)-\mu(i) & \text { if } i=j,
\end{array} \quad D_{1}(i, j)=\left\{\begin{array}{cl}
Q(i, j) \mu(i, j) & \text { if } i \neq j, \\
\mu(i) & \text { if } i=j .
\end{array}\right.\right.
$$

Furthermore, we have $Q^{*}=Q$ and we retrieve the fact that failures do not affect the execution process.

\section{Example 2.2 (Markov modulated Poisson Process)}

Another interesting point process is the one obtained by assuming in Littlewood's model that the probability of a secondary failure during a control transfer is 0 . In this case, setting $\mu(i, j)=0$ for all $i, j \in \mathcal{M}$ in the previous expressions, we get $D_{0}=Q-\operatorname{diag}(\mu(i))$ and $D_{1}=\operatorname{diag}(\mu(i))$. This an MMPP.

Note that $Z^{\prime}=\left(X_{t}^{*}, N_{t}\right)_{t}$ is also a Markov process with homogeneous second component as defined in [5], or a Markov-additive process of arrivals discussed in [16].

\subsection{Compensator and intensity of the counting process}

The basic facts on point processes, martingales, compensator and intensity used in this paper are reported in [2]. A nice survey on point processes is [17]. For any process $V=\left(V_{t}\right)_{t}, \mathcal{F}^{V}=\left(\mathcal{F}_{t}^{V}\right)_{t}$ will denote its internal history, i.e. $\mathcal{F}_{t}^{V}:=\sigma\left(V_{s}, s \leq t\right)$.

Considering the bivariate process $Z$ to analyze the counting process $N$ allows to deal with a Markov process with discrete state space. Thus, we can take advantage of the powerful analytic theory and the computational material developed for such class of processes. This fact was exploited in [10],[9] to assess various reliability measures related to the transient behavior of the counting process. Due to the special structure of the 
generator $G$ of $Z, N$ may be interpreted as the counter of specific transitions in $Z$. More precisely, if we are interested in the following pairs of states in $S$

$$
T:=\{((n, i) ;(n+1, j)), \quad i, j \in \mathcal{M}, n \geq 0\}
$$

then

$$
\begin{aligned}
N_{t} & =\sum_{(x, y) \in T} \sum_{0<s \leq t} \mathbf{1}_{\left\{Z_{s-}=x, Z_{s}=y\right\}} \\
& =\sum_{(x, y) \in T} N_{t}(x, y) .
\end{aligned}
$$

In other words, $N_{t}$ counts the transitions from state $x$ to $y(x, y \in T)$ in interval $\left.] 0, t\right]$. It is well-known (see e.g. [2]) that for a counter $N(x, y)=\left(N_{t}(x, y)\right)_{t}$ of transitions from state $x$ to $y$ in a Markov chain, we have

$$
N_{t}(x, y)-\int_{0}^{t} \mathbf{1}_{\left\{Z_{s-}=x\right\}} G(x, y) d s
$$

is a $\mathcal{F}^{Z}=\left(\mathcal{F}_{t}^{Z}\right)_{t}$ martingale. The random function

$$
\lambda_{t}(x, y):=\mathbf{1}_{\left\{Z_{t-}=x\right\}} G(x, y)
$$

is the $\mathcal{F}^{Z}$-intensity of $N(x, y)$ and

$$
A_{t}(x, y):=\int_{0}^{t} \lambda_{s}(x, y) d s
$$

is the $\mathcal{F}^{Z}$-compensator $\left(\mathcal{F}_{t}^{Z}\right.$ dual predictable-projection) of $N(x, y)$. Then it is easily seen that $M=\left(M_{t}\right)_{t}$ with $M_{t}=N_{t}-A_{t}$ and

$$
A_{t}=\sum_{(x, y) \in T} A_{t}(x, y)
$$

is a $\mathcal{F}^{Z}$-martingale. The $\mathcal{F}^{Z}$-intensity of $N$ is

$$
\begin{aligned}
\lambda_{t} & :=\sum_{(x, y) \in T} \mathbf{1}_{\left\{Z_{s-}=x\right\}} G(x, y) \\
& =\sum_{n \geq 0} \sum_{i \in \mathcal{M}} \sum_{j \in \mathcal{M}} G((n, i) ;(n+1, j)) \mathbf{1}_{\left\{\left(N_{t-}, X_{t-}^{*}\right)=(n, i)\right\}} \\
& =\sum_{n \geq 0} \sum_{i \in \mathcal{M}} \sum_{j \in \mathcal{M}} D_{1}(i, j) \mathbf{1}_{\left\{\left(N_{t-}, X_{t-}^{*}\right)=(n, i)\right\}} \\
& =\sum_{i \in \mathcal{M}} \mathbf{1}_{\left\{X_{t-}^{*}=i\right\}}\left[\sum_{j \in \mathcal{M}} D_{1}(i, j)\right]=\sum_{j \in \mathcal{M}} D_{1}\left(X_{t-}^{*}, j\right) .
\end{aligned}
$$


In the case of Example 2.1, the intensity of the counting process corresponding to Littlewood's model is

$$
\mu\left(X_{t-}\right)+\sum_{j \neq X_{t-}} Q\left(X_{t-}, j\right) \mu\left(X_{t-}, j\right) .
$$

In the case of Example 2.2, we retrieve the well-known expression for the $\mathcal{F}^{(N, X)}$ intensity for an MMPP

$$
\mu\left(X_{t-}\right)
$$

\section{Convergence of the counting process}

\subsection{What does it mean that failure parameters are small?}

A basic way to represent smaller and smaller failure parameters is to multiply each of them by a scalar $\varepsilon$ and to investigate the behavior of the counting process $N$ as $\varepsilon$ tends to 0 . Thus, we consider the perturbated failure parameters

$$
\varepsilon \lambda(i), \varepsilon \lambda(i, j), \varepsilon \mu(i), \varepsilon \mu(i, j), \quad i, j \in \mathcal{M} \text {. }
$$

Let us consider the example of an MMPP (see Example 2.2) with associated matrices

$$
Q=\left(\begin{array}{cc}
-1 & 1 \\
1 & -1
\end{array}\right) \quad D_{1}=\left(\begin{array}{cc}
\mu(1) & 0 \\
0 & \mu(2)
\end{array}\right)
$$

$(\mu(1) \neq \mu(2))$ and with $\alpha=\pi=(1 / 2,1 / 2)$ (i.e. with a stationary environment). Matrix $D_{0}^{(\varepsilon)}$ is

$$
D_{0}^{(\varepsilon)}=Q-\left(\begin{array}{cc}
\mu(1) \varepsilon & 0 \\
0 & \mu(2) \varepsilon
\end{array}\right)
$$

If $T$ is the time to first failure, then

$$
\mathbb{P}\{T>t\}=\mathbb{P}\left\{N_{t}=0\right\}=\pi \exp \left(D_{0}^{(\varepsilon)} t\right) \mathbf{1}^{\mathrm{t}} .
$$

Setting $a=4+(\mu(1)-\mu(2))^{2} \varepsilon^{2}$, we have

$$
\begin{aligned}
\pi \exp \left(D_{0}^{(\varepsilon)} t\right) \mathbf{1}^{\mathrm{t}}= & {\left[\cosh \left(\frac{t \sqrt{a}}{2}\right)+\frac{2}{\sqrt{a}} \sinh \left(\frac{t \sqrt{a}}{2}\right)\right] \exp (-t) } \\
& \times \exp (-(\mu(1) \pi(1)+\mu(2) \pi(2)) \varepsilon t)
\end{aligned}
$$

As it is expected, $\mathbb{P}\{T>t\}$ converges to 1 as $\varepsilon$ tends to 0 . Therefore, convergence in distribution of the counting process to an HPP, i.e. weak convergence of the finitedimensional distributions of $N$ to those of an HPP, can not take place at the current time 
scale. If we investigate the asymptotic distribution of $T$ at time scale $t / \varepsilon$, we get from previous expression for $\mathbb{P}\{T>t\}$

$$
\lim _{\varepsilon \rightarrow 0} \mathbb{P}\{T>t / \varepsilon\}=\exp (-(\mu(1) \pi(1)+\mu(2) \pi(2)) t) .
$$

Therefore, we will deal with the counting process $N^{(\varepsilon)}=\left(N_{t}^{(\varepsilon)}\right)_{t}$ defined by

$$
N_{t}^{(\varepsilon)}=N_{\frac{t}{\varepsilon}}
$$

where $N_{t}$ counts the number of failures in interval ] $\left.0, t\right]$ for the reliability model of Section 2 with system (3.1) of perturbated failure parameters. $D_{0}^{(\varepsilon)}, D_{1}^{(\varepsilon)}$ are the matrices associated with model $N$. Note that

$$
\begin{gathered}
D_{1}^{(\varepsilon)}=\varepsilon B+\varepsilon^{2} L \\
\text { with } L(i, j)= \begin{cases}-Q(i, j) \lambda(i, j) \mu(i, j) & \text { if } j \neq i \\
0 & \text { if } j=i\end{cases} \\
B(i, j)= \begin{cases}{\left[\lambda(i)+\sum_{k \neq i} Q(i, k) \lambda(i, k)\right] p(i, j)+Q(i, j) \mu(i, j)} & \text { if } j \neq i \\
D_{1}(i, i) & \text { if } j=i\end{cases}
\end{gathered}
$$

Note that $B$ is a nonnegative matrix. The Markov processes $X^{*}:=\left(X_{t}^{*}\right)_{t}$ and $X^{*, \varepsilon}:=$ $\left(X_{t / \varepsilon}^{*}\right)_{t}$ have as generator $Q^{*}=D_{0}^{(\varepsilon)}+D_{1}^{(\varepsilon)}$ and $Q^{*, \varepsilon}:=Q^{*} / \varepsilon$ respectively.

\subsection{Convergence of compensators}

Using development of Subsection 2.2, the $\mathcal{F}^{\left(N^{(\varepsilon)}, X^{*, \varepsilon}\right)}$-compensator of $N^{(\varepsilon)}$ is

$$
\begin{aligned}
A_{t}^{(\varepsilon)} & =\int_{0}^{t / \varepsilon} \sum_{j \in \mathcal{M}} D_{1}^{(\varepsilon)}\left(X_{s-}^{*}, j\right) d s \\
& =\varepsilon \int_{0}^{t / \varepsilon} \sum_{j \in \mathcal{M}} B\left(X_{s-}^{*}, j\right) d s+\varepsilon^{2} \int_{0}^{t / \varepsilon} \sum_{j \in \mathcal{M}} L\left(X_{s-}^{*}, j\right) d s \quad \text { from }(3.2) \\
& =\sum_{i \in \mathcal{M}} \int_{0}^{t / \varepsilon} \varepsilon \mathbf{1}_{\left\{X_{s-}^{*}=i\right\}} d s \sum_{j \in \mathcal{M}} B(i, j)+\sum_{i \in \mathcal{M}} \int_{0}^{t / \varepsilon} \varepsilon^{2} \mathbf{1}_{\left\{X_{s-}^{*}=i\right\}} d s \sum_{j \in \mathcal{M}} L(i, j) .
\end{aligned}
$$

Since $X^{*}$ is c.a.d-l.a.g., we have for almost all $\omega \in \Omega, X_{s}^{*}(\omega)=X_{s-}^{*}(\omega)$ except for countably many $s$. Thus, we can replace $X_{s-}^{*}$ by $X_{s}^{*}$ in the above integrals. This observation will be used to equate similar integrals throughout this paper.

Now, let us consider the Littlewood's model corresponding to Example 2.1. Failures do not affect the execution process, that is $X^{*}=X$ and $Q^{*}=Q$. Then

$$
A_{t}^{(\varepsilon)}=\sum_{i \in \mathcal{M}} \varepsilon \int_{0}^{t / \varepsilon} \mathbf{1}_{\left\{X_{s}=i\right\}} d s \sum_{j \in \mathcal{M}} B(i, j) .
$$


It follows from well-known time-average properties of cumulative process $\int_{0}^{t} f\left(X_{s}\right) d s$ for an irreducible Markov process $X$ (see [3]) that $(\varepsilon / t) \int_{0}^{t / \varepsilon} \mathbf{1}_{\left\{X_{s}=i\right\}} d s$ converges a.s. to $\pi(i)$ where $\pi$ is the stationary distribution of $X$. Thus, we derive that

$$
A_{t}^{(\varepsilon)} \underset{\varepsilon \rightarrow 0}{\stackrel{a . s .}{\longrightarrow}} t \sum_{i \in \mathcal{M}} \pi(i) \sum_{j \in \mathcal{M}} B(i, j)=\lambda t
$$

with $\lambda$ as in (1). In particular this implies the probability convergence of $A_{t}^{(\varepsilon)}$ to $\lambda t$. We recognize the compensator of an HPP with intensity $\lambda$. It follows from $[7$, Th 1$]$ that

$$
N^{(\varepsilon)} \underset{\varepsilon \rightarrow 0}{\stackrel{d}{\longrightarrow}} P
$$

where $P=\left(P_{t}\right)_{t}$ is the counting process of an HPP with parameter $\lambda$. We have shown that Theorem 3.1 holds for an MMPP or the Littlewood's reliability model (Examples 2.1,2.2).

Since convergence in $L^{2}$-norm implies convergence in probability, the following lemma will give the convergence in probability of compensator $A_{t}^{(\varepsilon)}$ to $\lambda t$ as $\varepsilon$ tends to 0 for the general reliability model of Section 2 .

Lemma 3.1 If probability vector $\pi$ is such that $\pi Q=0$, then

$$
\lim _{\varepsilon \rightarrow 0} E\left(\varepsilon \int_{0}^{t / \varepsilon}\left(\mathbf{1}_{\left\{X_{s}^{*}=i\right\}}-\pi(i)\right) d s\right)^{2}=0 .
$$

ProofT. he perturbated generator $Q^{*}=D_{0}^{(\varepsilon)}+D_{1}^{(\varepsilon)}$ of $X^{*}$ can be decomposed under the following form

$$
Q^{*}=Q+R \varepsilon
$$

$$
\text { with } R(i, j)= \begin{cases}{\left[\lambda(i)+\sum_{k \neq i} Q(i, k) \lambda(i, k)\right] p(i, j)-Q(i, j) \lambda(i, j)} & \text { if } j \neq i \\ {\left[\lambda(i)+\sum_{k \neq i} Q(i, k) \lambda(i, k)\right] p(i, i)-\lambda(i)} & \text { if } j=i\end{cases}
$$

Changes of variables $u=s \varepsilon$ gives that $\varepsilon \int_{0}^{t / \varepsilon} \mathbf{1}_{\left\{X_{s}^{*}=i\right\}} d s=\int_{0}^{t} \mathbf{1}_{\left\{X_{s}^{*, \varepsilon}=i\right\}} d s$. Recall that $\left(X_{t}^{*, \varepsilon}\right)_{t}$ has $Q^{*} / \varepsilon=Q / \varepsilon+R$ as generator. It is easily checked $R \mathbb{I}^{\mathrm{t}}=0$ where $\mathbb{I}^{\mathrm{t}}$ is the $M$-dimensional column vector whose all entries are 1 . In such a case, [18, Corollary C.1,p 349] gives the estimate

$$
E\left|\int_{0}^{t}\left(\mathbf{1}_{\left\{X_{s}^{*, \varepsilon}=i\right\}}-\pi(i)\right) d s\right|^{2} \leq C\left(1+t^{2}\right) \varepsilon .
$$

The convergence of $\int_{0}^{t} \mathbf{1}_{\left\{X_{s}^{*, \varepsilon}=i\right\}} d s$ to $\pi(i)$ in $L^{2}$-norm as $\varepsilon$ tends to 0 , follows from the previous estimate.

The following theorem follows from [7, Th 1]. 
Theorem 3.1 Probability vector $\pi$ is such that $\pi Q=0$. As $\varepsilon$ tends to 0 , the counting process $N^{(\varepsilon)}=\left(N_{t / \varepsilon}\right)_{t}$ converges in distribution to the counting process of an HPP with intensity

$$
\lambda=\sum_{i \in \mathcal{M}} \pi(i)\left[\mu(i)+\lambda(i)+\sum_{j \neq i} Q(i, j)[\mu(i, j)+\lambda(i, j)]\right] .
$$

\subsection{Fast modulation in MMPP}

When we consider an MMPP (see Example 2.2), the previous issue is equivalent to investigate the asymptotic of a Poisson process with an intensity modulated by a fast Markov chain. Indeed, the compensator of $N^{(\varepsilon)}$ is then

$$
\begin{aligned}
A_{t}^{(\varepsilon)} & =\varepsilon \int_{0}^{t / \varepsilon} \mu\left(X_{s-}\right) d s \\
& =\int_{0}^{t} \mu\left(X_{\frac{s}{\varepsilon}-}\right) d s \quad \text { with changes of variable } u=\varepsilon s
\end{aligned}
$$

The Markov chain $X^{(\varepsilon)}=\left(X_{t / \varepsilon}\right)_{t}$ has the generator $Q / \varepsilon$. As $\varepsilon$ tends to 0 , it is now clear that introduction of small parameter $\varepsilon$ speeds up the rate of switches between states. Theorem 3.1 states that the asymptotic process is an HPP with intensity

$$
\lambda=\sum_{i \in \mathcal{M}} \pi(i) \mu(i)
$$

This fact is known and has been investigated for various applications. The closest context of reliability theory is [1], where the underlying Poisson process is either homogeneous or nonhomogeneous. Proofs are based on asymptotic expansion of the transition semigroup of the bivariate Markov process $Z^{(\varepsilon)}$. Introduction of time-dependent intensity is not relevant in our context. Indeed, the decrease of failure parameters, or the reliability growth, is already taken into account by the small parameter $\varepsilon$.

The case where the modulating process is a finite nonhomogeneous Markov process $X$ is addressed in [4]. The asymptotic process is a nonhomogeneous Poisson process with suitable ergodicity assumptions on $X$.

From a strictly mathematical point of view, the issue addressed in this paper is equivalent to both a fast modulating MP $X^{*}$ (with generator $Q^{*} / \varepsilon$ ) and the introduction of a small scalar $\varepsilon$ only in the failure parameters $\mu(\cdot, \cdot)$ corresponding to a transition between states. Speed up the modulating MP $X^{*}$ at rate $1 / \varepsilon$ implies a speeding up of the number of transitions between states of $X^{*}$. Therefore, we have to compensate this "explosion" by introducing a small factor $\varepsilon$ in $\mu(\cdot, \cdot)$. 


\section{Convergence rate}

We provide in this section an estimate of the convergence rate of the finite-dimensional distributions of $N^{(\varepsilon)}$ to those of an HPP with intensity as in Theorem 3.1. The counting process of the HPP is denoted by $P=\left(P_{t}\right)_{t}$. Note that $\lambda$ in (3.5) is the scalar product $\left\langle\pi, B \mathbb{I}^{\mathrm{t}}\right\rangle$, where $\mathbb{I}^{\mathrm{t}}$ is the $M$-dimensional column vector whose all entries are 1 . We can write the limit compensator as

$$
A_{t}=\left\langle\pi, B \mathbb{1}^{\mathrm{t}}\right\rangle t
$$

The $\mathcal{F}^{N^{(\varepsilon)}, X^{*, \varepsilon}}$-compensator of $N^{(\varepsilon)}$ is from (3.3)

$$
\begin{aligned}
A_{t}^{(\varepsilon)} & \left.=\sum_{i \in \mathcal{M}}(B+\varepsilon L) \mathbb{I}^{\mathrm{t}}\right)(i) \int_{0}^{t / \varepsilon} \varepsilon \mathbf{1}_{\left\{X_{s-}^{*}=i\right\}} d s \\
& \left.\left.=\sum_{i \in \mathcal{M}}(B+\varepsilon L) \mathbb{I}^{\mathrm{t}}\right)(i) \int_{0}^{t} \mathbf{1}_{\left\{X_{s-}^{*, \varepsilon}=i\right\}} d s \quad \text { (setting } u=s \varepsilon\right) \\
& =\int_{0}^{t}\left\langle Y_{s-}^{(\varepsilon)},(B+\varepsilon L) \mathbb{I}^{\mathrm{t}}\right\rangle d s
\end{aligned}
$$

where $Y_{s}^{(\varepsilon)}:=\left(\mathbf{1}_{\left\{X_{s}^{*, \varepsilon}=i\right\}}\right)_{i \in \mathcal{M}}$. Hence, the $\mathcal{F}^{N^{(\varepsilon)}, X^{*, \varepsilon}}$-intensity of $N^{(\varepsilon)}$ is

$$
\lambda_{s}^{(\varepsilon)}:=\left\langle Y_{s-}^{(\varepsilon)},(B+\varepsilon L) \mathbb{I}^{\mathrm{t}}\right\rangle
$$

Let $T$ be any positive scalar and $\mathbb{T}:=\left\{t_{0}, t_{1}, \ldots, t_{n}\right\}$ with $0=t_{0}<t_{1}<\cdots<$ $t_{n}=T$. To evaluate proximity between the respective distributions $\mathcal{L}\left(N_{\mathbb{T}}^{(\varepsilon)}\right)$ and $\mathcal{L}\left(P_{\mathbb{T}}\right)$ of $N_{\mathbb{T}}^{(\varepsilon)}:=\left(N_{t_{1}}^{(\varepsilon)}, \ldots, N_{t_{n}}^{(\varepsilon)}\right)$ and $P_{\mathbb{T}}:=\left(P_{t_{1}}, \ldots, P_{t_{n}}\right)$, the distance in total variation, denoted by $\mathrm{d}_{\mathrm{TV}}\left(\mathcal{L}\left(N_{\mathbb{T}}^{(\varepsilon)}\right), \mathcal{L}\left(P_{\mathbb{T}}\right)\right)$, may be used, that is $[17]$

$$
\begin{aligned}
\mathrm{d}_{\mathrm{TV}}\left(\mathcal{L}\left(N_{\mathbb{T}}^{(\varepsilon)}\right), \mathcal{L}\left(P_{\mathbb{T}}\right)\right) & \stackrel{\text { def }}{=} \sup _{B \subset \mathbb{N}^{n}}\left|\mathbb{P}\left\{N_{\mathbb{T}}^{(\varepsilon)} \in B\right\}-\mathbb{P}\left\{P_{\mathbb{T}} \in B\right\}\right| \\
& =\frac{1}{2} \sum_{\mathbf{k} \in \mathbb{N}^{n}}\left|\mathbb{P}\left\{N_{\mathbb{T}}^{(\varepsilon)}=\mathbf{k}\right\}-\mathbb{P}\left\{P_{\mathbb{T}}=\mathbf{k}\right\}\right|
\end{aligned}
$$

For a locally bounded variation function $t \mapsto f(t)$, the total variation in the interval $[0, T]$ is

$$
\operatorname{Var}_{[0, T]}(f) \stackrel{\text { def }}{=} \sup _{\left\{t_{1}, \ldots, t_{n}\right\} \in \mathcal{P}([0, T])} \sum_{i=1}^{n}\left|f\left(t_{i}\right)-f\left(t_{i-1}\right)\right|
$$

where $\mathcal{P}([0, T])$ is the set of all the finite subdivisions of the interval $[0, T]$.

In this section, we state that the finite-dimensional distributions of $N^{(\varepsilon)}$ converges in total variation to those of an HPP with intensity $\lambda$ at rate $\varepsilon$. Proof is borrowed from [8, Th 6.1] where a similar result is stated for an MMPP. This is heavily based on the 
following estimate of the total variation between finite-dimensional distributions of $N^{(\varepsilon)}$ and $P[8$, Th 3.1]

$$
\mathrm{d}_{\mathrm{TV}}\left(\mathcal{L}\left(N_{\mathbb{T}}^{(\varepsilon)}\right), \mathcal{L}\left(P_{\mathbb{T}}\right)\right) \leq E \operatorname{Var}_{[0, T]}\left(\widehat{A}^{(\varepsilon)}-A\right)
$$

where $\widehat{A}^{(\varepsilon)}$ is the $\mathcal{F}^{N^{(\varepsilon)}}$-compensator of $N^{(\varepsilon)}$. $\widehat{A}^{(\varepsilon)}$ is from (4.2) and [11, Th 18.3]

$$
\widehat{A}_{t}^{(\varepsilon)}=\int_{0}^{t}\left\langle\widehat{Y}_{s-}^{(\varepsilon)},(B+\varepsilon L) \mathbb{I}^{t}\right\rangle d s
$$

with $\widehat{Y}_{t}^{(\varepsilon)}:=\left(\mathbb{P}\left\{X_{t}^{*, \varepsilon}=i \mid \mathcal{F}_{t}^{N^{(\varepsilon)}}\right\}\right)_{i \in \mathcal{M}}$. Hence, the $\mathcal{F}^{N^{(\varepsilon)}}$-intensity of $N^{(\varepsilon)}$ is

$$
\widehat{\lambda}_{s}^{(\varepsilon)}:=\left\langle\widehat{Y}_{s-}^{(\varepsilon)},(B+\varepsilon L) \mathbb{I}^{\mathrm{t}}\right\rangle .
$$

Therefore, we obtain from (4.1) and (4.2) that

$$
\begin{aligned}
\mathrm{d}_{\mathrm{TV}}\left(\mathcal{L}\left(N_{\mathbb{T}}^{(\varepsilon)}\right), \mathcal{L}\left(P_{\mathbb{T}}\right)\right) \leq & E \operatorname{Var}_{[0, T]}\left(\int_{0}^{t}\left\langle\widehat{Y}_{s-}^{(\varepsilon)}-\pi, B \mathbb{I}^{\mathrm{t}}\right\rangle+\varepsilon\left\langle\widehat{Y}_{s-}^{(\varepsilon)}, L \mathbb{I}^{\mathrm{t}}\right\rangle d s\right) \\
& =E \int_{0}^{T}\left|\left\langle\widehat{Y}_{s-}^{(\varepsilon)}-\pi, B \mathbb{I}^{\mathrm{t}}\right\rangle+\varepsilon\left\langle\widehat{Y}_{s-}^{(\varepsilon)}, L \mathbb{I}^{\mathrm{t}}\right\rangle\right| d s \\
\leq & E \int_{0}^{T}\left|\left\langle\widehat{Y}_{s-}^{(\varepsilon)}-\pi, B \mathbb{I}^{\mathrm{t}}\right\rangle\right| d s+\varepsilon E \int_{0}^{T}\left|\left\langle\widehat{Y}_{s-}^{(\varepsilon)}, L \mathbb{I}^{\mathrm{t}}\right\rangle\right| d s \\
\leq & E \int_{0}^{T}\left|\left\langle\widehat{Y}_{s-}^{(\varepsilon)}-\pi, B \mathbb{I}^{\mathrm{t}}\right\rangle\right| d s+\varepsilon C_{T} \\
& \quad \text { (since } \widehat{Y}^{(\varepsilon)} \text { is bounded). }
\end{aligned}
$$

Since $\widehat{Y}_{s}^{(\varepsilon)}$ and $\pi$ are stochastic vectors, it follows from $(.19)$

$$
\left|\left\langle\widehat{Y}_{s}^{(\varepsilon)}-\pi, B \mathbb{I}^{\mathrm{t}}\right\rangle\right| \leq \frac{\delta}{2}\left\|\widehat{Y}_{s}^{(\varepsilon)}-\pi\right\|_{1}
$$

where $\delta:=\max \left(B \mathbb{I}^{\mathrm{t}}(i)\right)-\min \left(B \mathbb{1}^{\mathrm{t}}(i)\right)$ and $\|\cdot\|_{1}$ is the $l_{1}$-norm. Hence, it remains to estimate in (4.5) the convergence rate of $\left\|\widehat{Y}_{s}^{(\varepsilon)}-\pi\right\|_{1}$ to 0 when $\varepsilon \rightarrow 0$. The first step consists in writing a filtering equation for vector $Y_{t}^{(\varepsilon)}$.

\subsection{Filtering equation for $Y^{(\varepsilon)}$}

Let us recall that $Y_{t}^{(\varepsilon)}=\left(\mathbf{1}_{\left\{X_{t}^{*, \varepsilon}=i\right\}}\right)_{i \in \mathcal{M}}$. Note that each component $Y_{t}^{(\varepsilon)}(i)$ of vector $Y_{t}^{(\varepsilon)}$ is a bounded random variable. We basically follows [2, Ch IV].

Lemma 4.1 Define $\widehat{Y}_{t}^{(\varepsilon)}:=E\left[Y_{t}^{(\varepsilon)} \mid \mathcal{F}_{t}^{N^{(\varepsilon)}}\right]$. Let $\alpha$ be the probability distribution of $X_{0}^{* \varepsilon}$. We have for all $t \geq 0$

$$
\widehat{Y}_{t}^{(\varepsilon)}=\alpha+\frac{1}{\varepsilon} \int_{0}^{t} \widehat{Y}_{s-}^{(\varepsilon)} Q^{*} d s+\int_{0}^{t} v_{s-}^{(\varepsilon)}\left(d N_{s}^{(\varepsilon)}-\widehat{\lambda}_{s}^{(\varepsilon)} d s\right)
$$


where $\widehat{\lambda}^{(\varepsilon)}$ is the $\mathcal{F}^{N^{(\varepsilon)}}$-intensity of $N^{(\varepsilon)}$ given in (4.4) and

$$
v_{s-}^{(\varepsilon)}:=\frac{\widehat{Y}_{s-}^{(\varepsilon)}(B+\varepsilon L)}{\widehat{\lambda}_{s}^{(\varepsilon)}}-\widehat{Y}_{s-}^{(\varepsilon)} .
$$

Proof $W$. e recall that the MP $X^{*, \varepsilon}$ has the generator $Q^{*, \varepsilon}=Q^{*} / \varepsilon$. It follows from Dynkin formula that

$$
Y_{t}^{(\varepsilon)}=Y_{0}^{(\varepsilon)}+\frac{1}{\varepsilon} \int_{0}^{t} Y_{s-}^{(\varepsilon)} Q^{*} d s+M_{t}
$$

where $M=\left(M_{t}\right)$ is a $\mathcal{F}^{X^{*, \varepsilon}}$-martingale. Then applying [2, Ch IV, Th 1] to the representation (4.9) of the bounded process $Y^{(\varepsilon)}$, we get for $\widehat{Y}_{t}^{(\varepsilon)}$

$$
\widehat{Y}_{t}^{(\varepsilon)}=\widehat{Y}_{0}^{(\varepsilon)}+\frac{1}{\varepsilon} \int_{0}^{t} \widehat{Y}_{s-}^{(\varepsilon)} Q^{*} d s+\widehat{M}_{t}
$$

where $\widehat{M}=\left(\widehat{M}_{t}\right)$ is a $\mathcal{F}^{N^{(\varepsilon)}}$-martingale. Now, [2, Ch III, Th 17] gives us the following representation of the $\mathcal{F}^{N^{(\varepsilon)}}$-martingale $\widehat{M}$

$$
\int_{0}^{t} G_{s}\left(d N_{s}^{(\varepsilon)}-\widehat{\lambda}_{s}^{(\varepsilon)} d s\right)
$$

where $\widehat{\lambda}^{(\varepsilon)}$ is the $\mathcal{F}^{N^{(\varepsilon)}}$-intensity of $N^{(\varepsilon)}$ and $G=\left(G_{t}\right)$ is a $\mathcal{F}^{N^{(\varepsilon)}}$-predictable process called the innovations process. We also know from [2, Ch IV, Th 2] that $G_{t}:=G_{1, t}-\widehat{Y}_{t-}^{(\varepsilon)}+G_{3, t}$, where the entry $i$ of vectors $G_{1, s}$ and $G_{3, s}$ must be computed from

$$
\begin{aligned}
E \int_{0}^{t} C_{s} Y_{s}(i) \lambda_{s}^{(\varepsilon)} d s & =E \int_{0}^{t} C_{s} G_{1, s}(i) \hat{\lambda}_{s}^{(\varepsilon)} d s \\
E \sum_{0<s \leq t} C_{s} \Delta M_{s}(i) \Delta N_{s}^{(\varepsilon)} & =E \int_{0}^{t} C_{s} G_{3, s}(i) \hat{\lambda}_{s}^{(\varepsilon)} d s
\end{aligned}
$$

where $C:=\left(C_{s}\right)_{s}$ is any nonnegative $\mathcal{F}^{N^{(\varepsilon)}}$-predictable process, $\Delta M_{s}(i)=M_{s}(i)-M_{s-}(i)$ and $\Delta N_{s}^{(\varepsilon)}=N_{s}^{(\varepsilon)}-N_{s-}^{(\varepsilon)}$ are the jumps of martingale $M(i)$ and counting process $N^{(\varepsilon)}$, respectively. Random processes $\lambda^{(\varepsilon)}$ and $\widehat{\lambda}^{(\varepsilon)}$ are given by (4.3) and (4.4). We determine an explicit expression for $G_{1, t}$ and $G_{3, t}$. This is similar to the MMPP case (see [2, page $98]$ ) else but $X^{*, \varepsilon}$ and $N^{(\varepsilon)}$ may have common jumps. 
The left hand-side of Equality (4.10) can be rewritten as

$$
\begin{aligned}
E \int_{0}^{t} C_{s} Y_{s}^{(\varepsilon)}(i) \lambda_{s}^{(\varepsilon)} d s & =E \int_{0}^{t} C_{s} Y_{s-}^{(\varepsilon)}(i) \lambda_{s}^{(\varepsilon)} d s \\
& =E \int_{0}^{t} C_{s} Y_{s-}^{(\varepsilon)}(i)\left\langle Y_{s-}^{(\varepsilon)},(B+\varepsilon L) \mathbb{I}^{\mathrm{t}}\right\rangle d s \quad \text { from }(4.3) \\
& =E \int_{0}^{t} C_{s} Y_{s-}^{(\varepsilon)}(i)\left((B+\varepsilon L) \mathbb{I}^{\mathrm{t}}\right)(i) d s \\
& =E \int_{0}^{t} C_{s} \widehat{Y}_{s-}^{(\varepsilon)}(i)\left((B+\varepsilon L) \mathbb{I}^{\mathrm{t}}\right)(i) d s \text { since } C_{s} \text { is } \mathcal{F}_{s}^{N^{(\varepsilon)}} \text {-measurable. }
\end{aligned}
$$

Then, we deduce from (4.10) that

$$
G_{1, s}=\left(\frac{\widehat{Y}_{s-}^{(\varepsilon)}(i)\left((B+\varepsilon L) \mathbb{I}^{\mathrm{t}}\right)(i)}{\widehat{\lambda}_{s}^{(\varepsilon)}}\right)_{i \in \mathcal{M}}
$$

The counting process $N^{(\varepsilon)}$ has the following representation (see (2.1))

$$
N_{t}^{(\varepsilon)}=\sum_{n=0}^{+\infty} \sum_{j \in \mathcal{M}} \sum_{k \in \mathcal{M}} N_{t}^{(\varepsilon)}((n, j),(n+1, k)) .
$$

where $N_{t}^{(\varepsilon)}(x, y)$ is the cumulative number of transitions of the bivariate process $\left(N^{(\varepsilon)}, X^{*, \varepsilon}\right)$ up to time $t$. Since $\Delta M_{s}(i)=\Delta Y_{s}^{(\varepsilon)}(i)$, we have

$$
\sum_{0<s \leq t} C_{s} \Delta M_{s}(i) \Delta N_{s}^{(\varepsilon)}=\sum_{0<s \leq t} C_{s} Y_{s}^{(\varepsilon)}(i) \Delta N_{s}^{(\varepsilon)}-\sum_{0<s \leq t} C_{s} Y_{s-}^{(\varepsilon)}(i) \Delta N_{s}^{(\varepsilon)}
$$

The first term in the right-hand side of the last equality can be rewritten from (4.12)

$$
\sum_{0<s \leq t} C_{s} Y_{s}^{(\varepsilon)}(i) \Delta N_{s}^{(\varepsilon)}=\sum_{0<s \leq t} C_{s} Y_{s}^{(\varepsilon)}(i) \sum_{n=0}^{+\infty} \sum_{j \in \mathcal{M}} \sum_{k \in \mathcal{M}} \Delta N_{s}^{(\varepsilon)}((n, j),(n+1, k)) .
$$

It is easily seen that the right-hand side of the last equality is also

$$
\sum_{0<s \leq t} C_{s} \sum_{n=0}^{+\infty} \sum_{j \in \mathcal{M}} Y_{s-}^{(\varepsilon)}(j) \Delta N_{s}^{(\varepsilon)}((n, j),(n+1, i)) .
$$

So, we have from (4.13) and the previous equality

$$
\sum_{0<s \leq t} C_{s} \Delta M_{s}(i) \Delta N_{s}^{(\varepsilon)}=\int_{0}^{t} C_{s} \sum_{n=0}^{+\infty} \sum_{j \in \mathcal{M}} Y_{s-}^{(\varepsilon)}(j) d N_{s}^{(\varepsilon)}((n, j),(n+1, i))-\int_{0}^{t} C_{s} Y_{s-}^{(\varepsilon)}(i) d N_{s}^{(\varepsilon)}
$$


Since the processes $C$ and $Y^{(\varepsilon)}$ are $\mathcal{F}^{N^{(\varepsilon)}, X^{*, \varepsilon}}$-predictable, we get from the definition of the compensator, the formulae (2.2) and (4.3)

$$
\begin{aligned}
& E \sum_{0<s \leq t} C_{s} \Delta M_{s}(i) \Delta N_{s}^{(\varepsilon)} \\
= & E \int_{0}^{t} C_{s}\left[\sum_{j \in \mathcal{M}} Y_{s-}^{(\varepsilon)}(j)(B(j, i)+\varepsilon L(j, i))-Y_{s-}^{(\varepsilon)}(i)\left\langle Y_{s-}^{(\varepsilon)},(B+\varepsilon L) \mathbb{I}^{\mathrm{t}}\right\rangle\right] d s \\
= & E \int_{0}^{t} C_{s}\left[\sum_{j \in \mathcal{M}} Y_{s-}^{(\varepsilon)}(j)(B(j, i)+\varepsilon L(j, i))-Y_{s-}^{(\varepsilon)}(i) \sum_{j \in \mathcal{M}}(B(i, j)+\varepsilon L(i, j))\right] d s \\
= & E \int_{0}^{t} C_{s}\left[\sum_{j \neq i} Y_{s-}^{(\varepsilon)}(j)(B(j, i)+\varepsilon L(j, i))-Y_{s-}^{(\varepsilon)}(i) \sum_{j \neq i}(B(i, j)+\varepsilon L(i, j))\right] d s \\
= & E \int_{0}^{t} C_{s}\left[\sum_{j \neq i} \widehat{Y}_{s-}^{(\varepsilon)}(j)(B(j, i)+\varepsilon L(j, i))-\widehat{Y}_{s-}^{(\varepsilon)}(i) \sum_{j \neq i}(B(i, j)+\varepsilon L(i, j))\right] d s \\
& \text { since } C_{s} \text { is } \mathcal{F}_{s}^{N^{(\varepsilon)}} \text {-measurable. }
\end{aligned}
$$

We get from (4.11) that

$$
G_{3, s}(i)=\frac{\sum_{j \neq i} \widehat{Y}_{s-}^{(\varepsilon)}(j)(B(j, i)+\varepsilon L(j, i))-\widehat{Y}_{s-}^{(\varepsilon)}(i) \sum_{j \neq i}(B(i, j)+\varepsilon L(i, j))}{\widehat{\lambda}_{s}^{(\varepsilon)}} .
$$

Consequently, the gain $G_{s}$ has the form reported in Lemma 4.1.

Lemma 4.2 Let $\alpha$ be the probability distribution of $X_{0}^{* \varepsilon}$. Then Equation (4.7) has the unique solution

$$
\widehat{Y}_{t}^{(\varepsilon)}=\alpha \exp \left(Q^{*} t / \varepsilon\right)+\int_{0}^{t} v_{s-}^{(\varepsilon)} \exp \left(Q^{*}(t-s) / \varepsilon\right)\left(d N_{s}^{(\varepsilon)}-\widehat{\lambda}_{s}^{(\varepsilon)} d s\right) .
$$

ProofF. irst, let us check that the right-hand side term of (4.14) is a solution of (4.7). It has the form $U_{t} V_{t}$ where

$$
\begin{aligned}
U_{t} & =\alpha+\int_{0}^{t} v_{s-}^{(\varepsilon)} \exp \left(-Q^{*} s / \varepsilon\right)\left(d N_{s}^{(\varepsilon)}-\widehat{\lambda}_{s}^{(\varepsilon)} d s\right) \\
V_{t} & =\exp \left(Q^{*} t / \varepsilon\right) .
\end{aligned}
$$

Using an integration by parts [2, T2, p 336], we obtain

$$
\begin{aligned}
U_{t} V_{t} & =U_{0} V_{0}+\int_{0}^{t} U_{s} d V_{s}+\int_{0}^{t} d U_{s} V_{s} \\
& =\alpha+\frac{1}{\varepsilon} \int_{0}^{t}\left(U_{s} V_{s}\right) Q^{*} d s+\int_{0}^{t} v_{s-}^{(\varepsilon)}\left(d N_{s}^{(\varepsilon)}-\widehat{\lambda}_{s}^{(\varepsilon)} d s\right) .
\end{aligned}
$$


Second, note that $\left(\widehat{Y}_{t}^{(\varepsilon)}-U_{t} V_{t}\right)_{t}$ is a solution of the homogeneous linear differential equation

$$
y(t)=\frac{1}{\varepsilon} \int_{0}^{t} y(s) Q^{*} d s
$$

with initial condition $y(0)=0$. Then, $\widehat{Y}_{t}^{(\varepsilon)}-U_{t} V_{t} \equiv 0$.

\subsection{Convergence rate}

Theorem 4.1 $P=\left(P_{t}\right)$ is the counting process of an HPP with intensity

$$
\lambda=\left\langle\pi, B \mathbb{1}^{\mathrm{t}}\right\rangle=\sum_{i \in \mathcal{M}} \pi(i)\left[\mu(i)+\lambda(i)+\sum_{j \neq i} Q(i, j)(\lambda(i, j)+\mu(i, j))\right]
$$

where $\pi$ is the probability distribution such that $\pi Q=0$. For any $T>0$, there exists a constant $C_{T}$ such that

$$
\mathrm{d}_{\mathrm{TV}}\left(\mathcal{L}\left(N_{\mathbb{T}}^{(\varepsilon)}\right), \mathcal{L}\left(P_{\mathbb{T}}\right)\right) \leq C_{T} \varepsilon
$$

ProofL. et us recall that (see (4.5) and (4.6))

$$
\mathrm{d}_{\mathrm{TV}}\left(\mathcal{L}\left(N_{\mathbb{T}}^{(\varepsilon)}\right), \mathcal{L}\left(P_{\mathbb{T}}\right)\right) \leq \frac{\delta}{2} E \int_{0}^{T}\left\|\widehat{Y}_{t}^{(\varepsilon)}-\pi\right\|_{1} d t+C_{1, T} \varepsilon .
$$

We just have to control the first term in the right-hand side of the inequality. Since $v_{s-}^{(\varepsilon)} \mathbb{I}^{\mathrm{t}}=0$, we can write from $(4.14)$

$$
\begin{aligned}
\widehat{Y}_{t}^{(\varepsilon)}-\pi= & \alpha \exp \left(Q^{*} t / \varepsilon\right)-\pi \\
& +\int_{0}^{t} v_{s-}^{(\varepsilon)}\left[\exp \left(Q^{*}(t-s) / \varepsilon\right)-\mathbb{I}^{\mathrm{t}} \pi\right]\left(d N_{s}^{(\varepsilon)}-\widehat{\lambda}_{s}^{(\varepsilon)} d s\right) .
\end{aligned}
$$

Using the triangle inequality, it is easily seen that

$$
\begin{aligned}
E \int_{0}^{T}\left\|\widehat{Y}_{t}^{(\varepsilon)}-\pi\right\|_{1} d t \leq & \int_{0}^{T}\left\|\alpha \exp \left(Q^{*} t / \varepsilon\right)-\pi\right\|_{1} d t \\
& +E \int_{0}^{T}\left\|\int_{0}^{t} v_{s-}^{(\varepsilon)}\left[\exp \left(Q^{*}(t-s) / \varepsilon\right)-\mathbb{I}^{\mathrm{t}} \pi\right]\left(d N_{s}^{(\varepsilon)}-\widehat{\lambda}_{s}^{(\varepsilon)} d s\right)\right\|_{1} d t .
\end{aligned}
$$

In a first step, let us consider the former term in the right hand side of the previous inequality. We have from (.18) with $Q^{(\varepsilon)}=Q^{*} / \varepsilon=Q / \varepsilon+R$ (see (3.4)) that for all $s \geq 0$

$$
\left\|\alpha \exp \left(Q^{*} t / \varepsilon\right)-\pi\right\|_{1} \leq C_{1}(\varepsilon+\exp (-\rho t / \varepsilon)) .
$$

Then

$$
\int_{0}^{T}\left\|\alpha \exp \left(Q^{*} t / \varepsilon\right)-\pi\right\|_{1} d t \leq\left(C_{1} T+\frac{1}{\rho}\right) \varepsilon=C_{2, T} \varepsilon
$$


In a second step, we have

$$
\begin{aligned}
& E\left\|\int_{0}^{t} v_{s-}^{(\varepsilon)}\left[\exp \left(Q^{*}(t-s) / \varepsilon\right)-\mathbb{I}^{\mathrm{t}} \pi\right]\left(d N_{s}^{(\varepsilon)}-\widehat{\lambda}_{s}^{(\varepsilon)} d s\right)\right\|_{1} \\
\leq & E \int_{0}^{t}\left\|v_{s-}^{(\varepsilon)}\left[\exp \left(Q^{*}(t-s) / \varepsilon\right)-\mathbb{I}^{\mathrm{t}} \pi\right]\right\|_{1}\left(d N_{s}^{(\varepsilon)}+\widehat{\lambda}_{s}^{(\varepsilon)} d s\right) \\
= & 2 E \int_{0}^{t}\left\|v_{s-}^{(\varepsilon)}\left[\exp \left(Q^{*}(t-s) / \varepsilon\right)-\mathbb{I}^{\mathrm{t}} \pi\right]\right\|_{1} \widehat{\lambda}_{s}^{(\varepsilon)} d s
\end{aligned}
$$

since $\hat{\lambda}_{s}^{(\varepsilon)}$ is the $\mathcal{F}^{N^{(\varepsilon)}}$-intensity of $N^{(\varepsilon)}$ and $v_{s-}^{(\varepsilon)}$ is $\mathcal{F}^{N^{(\varepsilon)}}$-predictable. We recall that $\|x M\|_{1} \leq\|x\|_{1}\|M\|_{1}$ for any vector $x$ and matrix $M$, where $\|M\|_{1}:=\max _{i}\left(\sum_{j}|M(i, j)|\right)$ is the 1-matrix norm. Now, we get

$$
\left\|v_{s-}^{(\varepsilon)}\left[\exp \left(Q^{*}(t-s) / \varepsilon\right)-\mathbb{I}^{\mathrm{t}} \pi\right]\right\|_{1} \leq\left\|v_{s-}^{(\varepsilon)}\right\|_{1}\left\|\exp \left(Q^{*}(t-s) / \varepsilon\right)-\mathbb{I}^{\mathrm{t}} \pi\right\|_{1} .
$$

It follows from (.17) that for all $s \leq t$

$$
\left\|\exp \left(Q^{*}(t-s) / \varepsilon\right)-\mathbb{1}^{\mathrm{t}} \pi\right\|_{1} \leq C_{2}(\varepsilon+\exp (-(t-s) \rho / \varepsilon)) .
$$

Since $v_{s-}^{(\varepsilon)}$ is the difference between two probability vectors, we have $\left\|v_{s-}^{(\varepsilon)}\right\|_{1} \leq 2$. Using (4.4) and $\left\|\widehat{Y}_{s-}^{(\varepsilon)}\right\|_{1}=1$, we get $\left\|v_{s-}^{(\varepsilon)}\right\|_{1} \widehat{\lambda}_{s}^{(\varepsilon)} \leq 2\left\|(B+\varepsilon L) \mathbb{I}^{\mathrm{t}}\right\|_{\infty}$. Then

$$
\left\|v_{s-}^{(\varepsilon)}\right\|_{1} \widehat{\lambda}_{s}^{(\varepsilon)} \leq C_{3}+C_{4} \varepsilon
$$

is uniformly bounded in $s$. Therefore, for all $t \geq 0$, we can write

$$
\begin{aligned}
E \int_{0}^{t}\left\|v_{s-}^{(\varepsilon)}\left[\exp \left(Q^{*}(t-s) / \varepsilon\right)-\mathbb{I}^{t} \pi\right]\right\|_{1} \widehat{\lambda}_{s}^{(\varepsilon)} d s & \leq\left(C_{5}+\varepsilon C_{6}\right) \int_{0}^{t}(\varepsilon+\exp (-(t-s) \rho / \varepsilon)) d s \\
& \leq\left(C_{5}+\varepsilon C_{6}\right)\left(t \varepsilon+C_{7} \varepsilon\right) \\
& \leq\left(C_{8} t+C_{9}\right) \varepsilon . \quad(\text { for } \varepsilon \leq 1)
\end{aligned}
$$

We deduce from the previous estimate (and Fubini's theorem) that the second term in the right-hand side of Inequality (4.15) is such that

$$
\begin{aligned}
& E \int_{0}^{T}\left\|\int_{0}^{t} v_{s-}^{(\varepsilon)}\left(\exp \left(Q^{*}(t-s) / \varepsilon\right)-\mathbb{I}^{\mathrm{t}} \pi\right)\left(d N_{s}^{(\varepsilon)}-\widehat{\lambda}_{s}^{(\varepsilon)} d s\right)\right\|_{1} d t \\
\leq & \int_{0}^{T}\left(C_{8} t+C_{9}\right) \varepsilon d t=C_{3, T} \varepsilon \quad(\text { for } \varepsilon \leq 1) .
\end{aligned}
$$

Theorem 4.1 follows from (4.15) with the last inequality and (4.16). 
Remark 4.1 With respect to Theorem 3.1, note that [7, Th 1] would give, in fact, convergence in distribution of the counting process $N^{(\varepsilon)}$ to Poisson process $P$ in the space of all counting processes, equipped with the Skorokhod topology. Moreover, convergence in variation also takes place in this space. Indeed, the distance in total variation over interval $[0, T]$ between distributions of $N^{(\varepsilon)}$ and $P$ is also bounded from above by $E \operatorname{Var}_{[0, T]}\left(\widehat{A}^{(\varepsilon)}-A\right)$ (see [8, Th 4.1]). Thus, it follows from Theorem 4.1 that the rate of convergence is $\varepsilon$.

Remark 4.2 The order of the convergence rate in Theorem 4.1 cannot be improved in general. This follows from [4, Section 5, Example 1], where the authors report a lower bound for the distance in variation, that has order 1 in $\varepsilon$ for a Poisson process modulated by a 2-states Markov process.

Estimate of convergence rate

Let us consider a generator $Q^{(\varepsilon)}=Q_{1}+Q_{2} / \varepsilon$ where $Q_{2}$ is assumed to be an irreducible generator. There exists a probability vector $\pi$ such that $\pi Q_{2}=0$. The following estimates are provided in [18, Lemma C.3, p 346]: for all $t \geq 0$ and any probability vector $\alpha$, there exists constants $K$ and $\rho>0$ such that

$$
\begin{aligned}
\left\|\exp \left(Q^{(\varepsilon)} t\right)-\mathbb{1}^{\mathrm{t}} \pi\right\|_{1} & \leq K(\varepsilon+\exp (-\rho t / \varepsilon)) \\
\left\|\alpha \exp \left(Q^{(\varepsilon)} t\right)-\pi\right\|_{1} & \leq K(\varepsilon+\exp (-\rho t / \varepsilon))
\end{aligned}
$$

and $\rho$ only depends upon $Q_{2}$

A simple inequality

Let $v$ be any $M$-dimensional vector. Any convex combinations $\left\langle u_{1}, v\right\rangle$ and $\left\langle u_{2}, v\right\rangle$ of scalars $(v(i), i=1, \ldots, M)$ are in the interval $[\min (v(i)), \max (v(i))]$, so that $\mid\left\langle u_{1}-\right.$ $\left.u_{2}, v\right\rangle \mid \leq \delta$ with $\delta:=\max (v(i))-\min (v(i))$.

Let $\alpha_{1}$ and $\alpha_{2}$ two stochastic vectors. Write $\alpha_{1}-\alpha_{2}=\left(\alpha_{1}-\alpha_{2}\right)^{+}-\left(\alpha_{1}-\alpha_{2}\right)^{-}$where $w^{+}=(\max (w(i), 0))_{i=1, \ldots, M}, w^{-}=-(\min (w(i), 0))_{i=1, \ldots, M}$. Since $\left(\alpha_{1}-\alpha_{2}\right) \mathbb{I}^{\mathrm{t}}=0$, we have $\left\|\alpha_{1}-\alpha_{2}\right\|_{1}=\left(\alpha_{1}-\alpha_{2}\right)^{+} \mathbb{I}^{\mathrm{t}}+\left(\alpha_{1}-\alpha_{2}\right)^{-} \mathbb{1}^{\mathrm{t}}=2\left(\alpha_{1}-\alpha_{2}\right)^{+} \mathbb{1}^{\mathrm{t}}=2\left(\alpha_{1}-\alpha_{2}\right)^{-} \mathbb{1}^{\mathrm{t}}$, where $\|\cdot\|_{1}$ is the $l_{1}$-norm. Then, we may write the vector $\alpha_{1}-\alpha_{2}$ as $\left(u_{1}-u_{2}\right)\left\|\alpha_{1}-\alpha_{2}\right\|_{1} / 2$ where $u_{1}, u_{2}$ are the stochastic vectors

$$
u_{1}=\frac{\left(\alpha_{1}-\alpha_{2}\right)^{+}}{\left(\alpha_{1}-\alpha_{2}\right)^{+} \mathbb{1}^{\mathrm{t}}} \quad u_{2}=\frac{\left(\alpha_{1}-\alpha_{2}\right)^{-}}{\left(\alpha_{1}-\alpha_{2}\right)^{-} \mathbb{1}^{\mathrm{t}}} .
$$

Next, it follows from the first part that

$$
\left|\left\langle\alpha_{1}-\alpha_{2}, v\right\rangle\right|=\frac{\left\|\alpha_{1}-\alpha_{2}\right\|_{1}}{2}\left|\left\langle u_{1}-u_{2}, v\right\rangle\right| \leq \frac{\delta}{2}\left\|\alpha_{1}-\alpha_{2}\right\|_{1} .
$$

\section{References}

[1] Anisimov V.V. And Sityuk V.N. (1980). Asymptotic behavior of inhomogeneous poisson flow with conductor function, dependent on a small parameter controlled by a markov chain. Cybernetics 15, 526-532. 
[2] Bremaud P. (1981). Point Processes and Queues. Springer.

[3] Çinlar E. (1975). Introduction to stochastic processes. Prentice-Hall, Inc., Englewood Cliffs, New Jersey.

[4] Di Masi G.B. And Kabanov Y. (1993). The strong convergence of two-scale stochastic systems and singular perturbations of filtering equations. J. of Mathematical Systems, Estimation, and Control 3, 207-224.

[5] Ezhov I.I. and Skorokhod A.V. (1969). Markov processes with homogeneous second component II. Theory Probab. Appl. 14, 652-667.

[6] Goseva-Popstojanova K. And Trivedi K.S. (2001). Architecture-based approach to reliability assessment of software systems. Performance Evaluation 45, 179-204.

[7] Kabanov Y.M, Liptser R.S. and Shiryaev A.N. (1980). Some limit theorems for simple point processes (martingale approach). Stochastics 3, 203-216.

[8] Kabanov Y.M., Liptser R.S. and Shiryaev A.N. (1983). Weak and strong convergence of the distributions of counting processes. Theory Probab. Appl. 28, 303-336.

[9] Ledoux J. (1999). Availability modeling of modular software. IEEE Trans. Rel. 48, 159-168.

[10] Ledoux J. And Rubino G. (1997). Simple formulae for counting processes in reliability models. Adv. in Appl. Probab. 29, 1018-1038.

[11] Liptser R.S And Shiryaev A.N. (2001). Statistics of random processes II. Springer.

[12] Littlewood B. (1975). A reliability model for systems with Markov structure. Appl. Statist. 24, 172-177.

[13] Littlewood B. (1979). Software reliability model for modular program structure. IEEE Trans. Rel. 28, 241-246.

[14] Narayana S., N. And Liu D. (1992). Local poissonification of the markovian arrival process. Stoch. Models 8, 87-129.

[15] Neuts, M. F. (1989). Structured Stochastic Matrices of $M / G / 1$ Type and Their Applications. Marcel Dekker Inc., New-York and Basel. 
[16] Pacheco A. and Prabhu N.U. (1995). Chapter 6: Markov-additive processes of arrivals. In Advances in Queueing Theory, Methods, and Open Problems. ed. J. Dshalalow. Probability and Stochastic Series. CRC Press pp. 167-194.

[17] Serfozo R.F. (1990). Chapter 1: Point processes. In Handbook in Operations research and Managment Science. ed. Heyman D.P. and Sobel M.J. Elsevier Science, North-Holland.

[18] Sethi S.P. And Zhang Q. (1994). Hierarchical Decision Making in Stochastic Manufacturing Systems. Birkhäuser, Boston. 